\title{
Application of Quotient Rings for Stability Analysis in Chemical Systems
}

\author{
S. Sauerbrei, A. Sensse, M. Eiswirth \\ Fritz-Haber-Institut der Max-Planck-Gesellschaft, \\ Faradayweg 4-6, D-14195 Berlin, Germany
}

July 11,2010

\begin{abstract}
Concepts from algebraic geometry (polynomial rings) can be used to determine analytically the stationary solutions in chemical reactions systems, more generally, systems of ordinary differential equations of polynomial form. The stability analysis via the Jacobian matrix often leads to complicated expressions which can hardly be analyzed. It is shown that these expressions can be simplified by forming quotient rings of the corresponding polynomial ring. The coefficients in the characteristic equation of the Jacobian can be represented by the normal forms obtained by generating the quotient rings so that their sign changes in dependence of a kinetic parameter and hence the stability can be determined. The procedure is illustrated using a well-known surface reaction.
\end{abstract}

Key words: Toric Geometry; Quotient Rings; Mass Action Kinetics; Chemical Reaction Networks; Stoichiometric Network Analysis

\section{Introduction}

Nonlinear phenomena, such as multistability and oscillations, occur in a large number of chemical as well as biochemical systems. The experimental results could often be reproduced with numerical solutions of corresponding kinetic models by direct integration and bifurcation analysis. However the numerical task is often rendered very tedious due to a large parameter space and because some rate constants may be unknown (or only known approximately).

Therefore analytical methods have been developed which allow the prediction of instabilities from the stoichiometric network corresponding to the reaction system under consideration. On the basis of stoichiometric network analysis (SNA) [1], and concepts from algebraic geometry [5, 9-13], the stationary solutions of a chemical reaction system can be determined. This is possible since in general chemical reaction systems have polynomial structure due to mass action rate law or can be transformed into polynomials by the introduction of auxiliary variables $[7,13]$. The procedure is defined as follows: the stoichiometric matrix $N$ and the flux vector $v$ comprising the reaction rates of the chemical reaction system give the kinetic equations. Additionally, the intersection of the kernel of the stoichiometric matrix with the reaction rate space gives a convex polyhedral cone containing the nonnegative stationary

*sonja@fhi-berlin.mpg.de 
reaction rates [1]. This cone is spanned by the so-called extreme currents. A convex combination of the currents defines each element of the cone. Hence, the flux vector $v$ can be expressed by the sum over the currents with convex coefficients $j_{i}$.

By generating a binomial ideal of the reaction rates and its Groebner basis a variable separation is possible which leads to the restrictive polynomials. Via the restrictive polynomials the convex coefficients $j_{i}$ can be solved and a further transformation gives the set of the parameterized stationary concentrations which represents a curve in the concentration space.

Usually, a stability analysis follows via the Jacobian matrix. But if the stationary concentrations are complicated expressions, the stability analysis will be very difficult. A simplification of the coefficients of the characteristic polynomial is possible by using quotient rings which eliminate the concentrations and reaction rates as much as possible [10]. With the resulting expressions, sign changes in the coefficients in dependence of the kinetic parameters can be detected. The existence of a Hopf bifurcation (and thus oscillations) can often be predicted from SNA alone [6], and its location in the kinetic parameter space can be found using toric geometry $[11,12]$. The derivation of multistability tends to be more difficult (for a procedure based on injectivity, see $[3,4]$ ), and a simplification of the resulting expressions (via quotient rings) is called for.

In order to illustrate the above-mentioned concepts, particularly the quotient rings, in detail we use an abstract Langmuir-Hinshelwood mechanism [8]. In section 2 we carry out the stability analysis, the algebraic concepts (and the notation) follow the book by Cox et al. [2]. As a real reaction system the well-known carbon monoxide oxidation is investigated by the same procedure in section 4 .

\section{Langmuir-Hinshelwood Mechanism}

Most surface reactions obey the well-known Langmuir-Hinshelwood (LH) mechanism: two educts have to adsorb on the surface before reacting.

$$
\begin{aligned}
X_{1}+\nu_{x_{1}} * & \leftrightarrows X_{1, a d} \\
X_{2}+\nu_{x_{2}} * & \leftrightarrows X_{2, a d} \\
X_{1, a d}+X_{2, a d} & \rightarrow\left(\nu_{x_{1}}+\nu_{x_{2}}\right) *+X_{4}
\end{aligned}
$$

where $*$ denotes a vacant surface site, $\nu_{x_{1}}$ and $\nu_{x_{2}}$ give the number of sites which are required for adsorption of the corresponding species $X_{1}$ and $X_{2}$. The product $X_{4}$ has to desorb sufficiently fast, otherwise self-poisoning of the catalyst occurs. In [6] an abstract model of an LH mechanism was shown which exhibits bistability, except for the degenerated case when the site requirements and adsorption kinetics of $X_{1}$ and $X_{2}$ are exactly the same [8]. Due to the desorption $X_{4}$ is not taken into account.

Setting $\nu_{x_{1}}=2, \nu_{x_{2}}=1, x_{3}=*$, and assuming constant pressures of $X_{1}$ and $X_{2}$, the pseudo reaction equations of this model are given by

$$
\begin{array}{rll}
X_{1} & \stackrel{k_{1}}{\longrightarrow} & 2 X_{3} \\
2 X_{3} & \stackrel{k_{2}}{\longrightarrow} & X_{1} \\
X_{2} & \stackrel{k_{3}}{\longrightarrow} & X_{3} \\
X_{3} & \stackrel{k_{4}}{\longrightarrow} & X_{2} \\
X_{1}+X_{2} & \stackrel{k_{5}}{\longrightarrow} & 3 X_{3}
\end{array}
$$

and can be represented by the network diagram in Fig. 1 (left). The stoichiometric matrix $N$, the flux vector $v(x, k)$ comprising the reaction rates $v_{j}$, and the kinetic matrix $\kappa$ of the chemical reaction system 


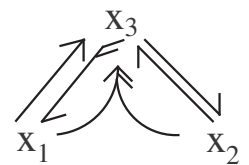

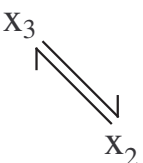

$\mathrm{E}_{1}$

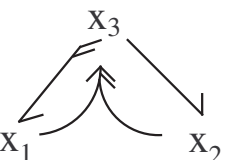

$\mathrm{E}_{2}$

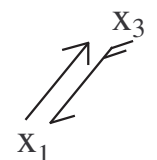

$\mathrm{E}_{3}$

Figure 1: Left: the network diagram of a Langmuir-Hinshelwood mechanism. $X_{1}, X_{2}$ represent the adsorbed species and $X_{3}$ denotes a vacant site. Right: the extreme currents $E_{i}$ of the Langmuir-Hinshelwood mechanism. $E_{1}$ and $E_{3}$ are equilibrium currents and hence stable. $E_{2}$ is unstable due to the higher site requirement of $X_{1}$. Note that $X_{4}$ is not taken into account since it has to desorb sufficiently fast.

in (1) are given by

$$
N=\left(\begin{array}{ccccc}
-1 & 1 & 0 & 0 & -1 \\
0 & 0 & -1 & 1 & -1 \\
2 & -2 & 1 & -1 & 3
\end{array}\right), v(x, k)=\left(\begin{array}{c}
k_{1} x_{1} \\
k_{2} x_{3}{ }^{2} \\
k_{3} x_{2} \\
k_{4} x_{3} \\
k_{5} x_{1} x_{2}
\end{array}\right), \kappa=\left(\begin{array}{ccccc}
1 & 0 & 0 & 0 & 1 \\
0 & 0 & 1 & 0 & 1 \\
0 & 2 & 0 & 1 & 0
\end{array}\right) .
$$

The reaction rates $v_{j}$ in $v(x, k)$ are modelled with a mass action rate law $v_{j}\left(k_{j}, x\right)=k_{j} \prod_{i=1}^{m} x^{\kappa_{i j}}$, whereby $\kappa_{i j}$ correspond to the kinetic exponents of species $i$ in the reaction $j$ which form kinetic matrix $\kappa$. Then the kinetic equations are given by $\dot{x}=N v(x, k)$ :

$$
\begin{aligned}
& \dot{x_{1}}=-k_{1} x_{1}+k_{2} x_{3}^{2}-k_{5} x_{1} x_{2} \\
& \dot{x_{2}}=-k_{3} x_{2}+k_{4} x_{3}-k_{5} x_{1} x_{2} \\
& \dot{x_{3}}=2 k_{1} x_{1}-2 k_{2} x_{3}^{2}+k_{3} x_{2}-k_{4} x_{3}+3 k_{5} x_{1} x_{2}
\end{aligned}
$$

with a conservation relation of the total number of adsorption sites:

$$
2 x_{1}+x_{2}+x_{3}=\text { const. }
$$

In the following sections the kinetic equations are solved via SNA and toric geometry. Note that we work in the reaction rate space at the beginning.

\subsection{Reduction of the Reaction Rate Space}

Clarke has shown that the nonnegative stationary reaction rates lie in a convex polyhedral cone $K_{v}$ [1]. This cone is obtained by the intersection of the kernel of the stoichiometric matrix $N$ (2) with the positive orthant of the $r$-dimensional reaction space $\mathbb{R}_{\geq 0}^{r}(r$ is the number of reactions):

$$
K_{v}=\left\{v \in \mathbb{R}^{r} \mid N v=0, v \geq 0\right\}=\left(\operatorname{ker} N \cap \mathbb{R}_{\geq 0}^{r}\right)=\left\{\sum_{i=1} j_{i} E_{i}, j_{i} \geq 0 \forall i\right\} .
$$

The cone is spanned by the extreme currents $E_{i}$. Each element within the cone can be represented as a nonnegative linear combination of the currents.

The stoichiometric matrix $N$ in (2) exhibits three extreme currents (Fig.1 on the right):

$$
E_{1}=\left(\begin{array}{l}
0 \\
0 \\
1 \\
1 \\
0
\end{array}\right), E_{2}=\left(\begin{array}{l}
0 \\
1 \\
0 \\
1 \\
1
\end{array}\right), E_{3}=\left(\begin{array}{l}
1 \\
1 \\
0 \\
0 \\
0
\end{array}\right)
$$


Then the flux vector can be expressed by the convex combination of the currents: $v(j)=\sum_{i=1}^{t} j_{i} E_{i}$ :

$$
v(j)=\left(\begin{array}{c}
j_{3} \\
j_{2}+j_{3} \\
j_{1} \\
j_{1}+j_{2} \\
j_{2}
\end{array}\right) .
$$

\subsection{Restrictive Polynomials}

The mentioned current cone contains all physically possible solutions for the complete parameter set. It is therefore desirable to pick from it the solutions generated by a given set of parameters $k_{i}$. Generally a system of $r$ reactions, $n$ species, $d$ of which are independent exhibits an $(r-d)$-dimensional current cone and $n-d$ conservation constraints. For "well-behaved" dissipative systems (which contain a countable number of stationary solutions, rather than a continuity) one therefore expects to find $r-n$ restrictive polynomials.

A polynomial ideal is generated by the monomial reaction rates $v_{j}(x, k)$ which are rewritten in a binomial form $v_{j}-v_{j}(x, k)$ and span the toric ideal

$$
J=\left\langle v_{1}-k_{1} x_{1}, v_{2}-k_{2} x_{3}^{2}, v_{3}-k_{3} x_{2}, v_{4}-k_{4} x_{3}, v_{5}-k_{5} x_{1} x_{2}\right\rangle .
$$

The set of zeros of this ideal is called toric variety. Note that the set of zeros of the basis elements of $J$ is equal to the set of zeros of the whole ideal $J$.

The restrictive polynomials are the result of a basis change of the ideal $J$. The transformation via a Groebner basis $(G B)$ leads to separation of variables. Since we are working in the reaction rate space we like to separate the reaction rates $v_{j}$ from the concentrations $x_{i}$. This requires the choice of an elimination order. To obtain the following Groebner basis $G B_{J}$ we choose the pure lexicographic order

$$
G B_{J}=\left\{-\mathbf{k}_{\mathbf{2}} \mathbf{v}_{\mathbf{4}}{ }^{\mathbf{2}}+\mathbf{k}_{\mathbf{4}}{ }^{\mathbf{2}} \mathbf{v}_{\mathbf{2}}, \mathbf{v}_{\mathbf{3}} \mathbf{v}_{\mathbf{1}} \mathbf{k}_{\mathbf{5}}-\mathbf{k}_{\mathbf{3}} \mathbf{k}_{\mathbf{1}} \mathbf{v}_{\mathbf{5}},-v_{4}+k_{4} x_{3},-v_{3}+k_{3} x_{2},-v_{1}+k_{1} x_{1}\right\}
$$

with variables ordering

$$
\left\{x_{1}, x_{2}, x_{3}, v_{1}, v_{2}, v_{3}, v_{4}, v_{5}\right\} .
$$

Note that the toric variety is not affected by the choice of the basis due to Hilbert's basis theorem. The concentrations are eliminated in the first two polynomials in (8) (bold) which represent the restrictive polynomials or the deformed toric variety. The reaction rates are confined to both the deformed toric variety and the cone $K_{v}$. Hence, the nonnegative reaction rates must lie in the intersection of the deformed toric variety and the cone

$$
V\left(I^{\text {def,tor }}\right) \cap K_{v}
$$

Since there is only one conservation relation between the variables the result of this intersection is a curve [9]: Substituting the reaction rates in convex coordinates (6) the restrictive polynomials are:

$$
\begin{aligned}
& 0=j_{1} j_{3} k_{5}-k_{1} k_{3} j_{2} \\
& 0=k_{4}^{2}\left(j_{2}+j_{3}\right)-k_{2}\left(j_{1}+2 j_{2}\right)^{2} .
\end{aligned}
$$

By arbitrarily choosing one of the convex coordinates as curve parameter $j$, the other coordinates are 
given by:

$$
\begin{aligned}
& j_{1}=1 / 2 \frac{\left(j k_{5} k_{4}+\sqrt{j^{2} k_{5}{ }^{2} k_{4}{ }^{2}+8 k_{2} k_{3} k_{1} j^{2} k_{5}+4 k_{2} j^{3} k_{5}{ }^{2}+4 j k_{2} k_{3}{ }^{2} k_{1}{ }^{2}}\right) k_{1} k_{3} k_{4}}{k_{2}\left(2 k_{3} k_{1} j k_{5}+j^{2} k_{5}{ }^{2}+k_{3}{ }^{2} k_{1}{ }^{2}\right)} \\
& j_{2}=1 / 2 \frac{\left(j k_{5} k_{4}+\sqrt{j^{2} k_{5}{ }^{2} k_{4}{ }^{2}+8 k_{2} k_{3} k_{1} j^{2} k_{5}+4 k_{2} j^{3} k_{5}{ }^{2}+4 j k_{2} k_{3}{ }^{2} k_{1}{ }^{2}}\right) k_{4} j k_{5}}{k_{2}\left(2 k_{3} k_{1} j k_{5}+j^{2} k_{5}{ }^{2}+{k_{3}}^{2}{k_{1}}^{2}\right)} \\
& j_{3}=j .
\end{aligned}
$$

The curve of the deformed toric variety in the space of the reaction rates is related to the set of nonnegative stationary concentrations via a special mapping (this a property of the intersection in (10)).

The set of stationary concentrations which results

$$
\left\{x \in \mathbb{R}_{\geq 0}^{m} \mid \exists k_{i}>0 \quad \text { with } \quad N v(x, k)=0\right\}
$$

is mapped onto the intersection set of the deformed toric variety and the cone

$$
\left\{v \mid v \in V\left(I^{\text {def,tor }}\right), v \geq 0, N v=0\right\}
$$

In one direction the mapping is given by $v: x \mapsto v(x, k)$. The inverse mapping (from the intersection curve to the set of stationary concentrations) is $v^{-1}: v\left(j_{i}, k\right) \mapsto x\left(j_{i}, k\right)$ and can be calculated by the Hermite normal form algorithm. For details and proofs of this relationship see [10,11].

In general, the Hermite normal form $H$ of a rectangular matrix of integers $A$ is its reduced echelon form. It holds that $U A=H$. For our purpose the Hermite normal form $H$ of the kinetic matrix $\kappa(2)$ is required: $U \kappa=H$, (16). Then the matrix $U$ contains the exponents of certain slack variables $w$ which will form monomials substituting the variables $x$, i.e. $x(w)$ (17). Thereby it is possible to represent the reaction rates in the slack variables too, $v(w)(17)$.

$$
\begin{aligned}
& \left(\begin{array}{lll}
1 & 0 & 0 \\
0 & 0 & 1 \\
0 & 1 & 0
\end{array}\right)\left(\begin{array}{lllll}
1 & 0 & 0 & 0 & 1 \\
0 & 0 & 1 & 0 & 1 \\
0 & 2 & 0 & 1 & 0
\end{array}\right)=\left(\begin{array}{lllll}
1 & 0 & 0 & 0 & 1 \\
0 & 2 & 0 & 1 & 0 \\
0 & 0 & 1 & 0 & 1
\end{array}\right), \\
& x(w)=\left(\begin{array}{c}
w_{1} \\
w_{3} \\
w_{2}
\end{array}\right), \quad v(w)=\left(\begin{array}{c}
k_{1} w_{1} \\
k_{2} w_{2}^{2} \\
k_{3} w_{3} \\
k_{4} w_{2} \\
k_{5} w_{1} w_{3}
\end{array}\right) .
\end{aligned}
$$

By setting $v(w)=v(j, k)$ the slack variables in dependence of the curve parameter $w(j, k)$ can be calculated and resubstituted into $x(w)$. Note that $v(j, k)$ is the result of (6) and (13). Then with the slack variables $w_{1}=j_{3} / k_{1}=j_{2} / k_{5} w_{3}, w_{2}=\sqrt{\left(j_{2}+j_{3}\right) / k_{2}}=\left(j_{1}+j_{2}\right) / k_{4}$, and $w_{3}=j_{1} / k_{3}=j_{2} / k_{5} w_{1}$ the set of stationary concentrations in dependence of the curve parameter $x(j, k)$ is given by:

$$
x(j, k)=\left(\begin{array}{c}
\frac{j}{k_{1}} \\
1 / 2 \frac{\left(j k_{5} k_{4}+\sqrt{j^{2} k_{5}{ }^{2} k_{4}{ }^{2}+8 k_{2} k_{3} k_{1} j^{2} k_{5}+4 k_{2} j^{3} k_{5}{ }^{2}+4 j k_{2} k_{3}{ }^{2} k_{1}^{2}}\right) k_{1} k_{4}}{k_{2}\left(2 k_{3} k_{1} j k_{5}+j^{2} k_{5}{ }^{2}+k_{3}{ }^{2} k_{1}{ }^{2}\right)} \\
1 / 2 \frac{j k_{5} k_{4}+\sqrt{j\left(j k_{5}{ }^{2} k_{4}{ }^{2}+8 k_{2} k_{3} k_{1} j k_{5}+4 k_{2} j^{2} k_{5}{ }^{2}+4 k_{2} k_{3}{ }^{2} k_{1}{ }^{2}\right)}}{\left(k_{3} k_{1}+j k_{5}\right) k_{2}}
\end{array}\right)
$$




\section{Stability Analysis}

The stationary concentrations (18) could be calculated analytically. However, the expressions appear quite complicated. The stability analysis via the Jacobian matrix in the phase space seems to be impossible, because of the radical expressions in the stationary concentrations (18). Nevertheless, the characteristic polynomial and the Hurwitz determinants in general represent polynomials in the variables $x$. So one may expect some advantages from using elimination theory once again. Actually, the appearing polynomials can be efficiently reduced evaluating them with quotient rings.

\subsection{Jacobian Matrix}

The Jacobian matrix is given by

$$
\operatorname{Jac}(k, x)=\left(\begin{array}{ccc}
-k_{1}-k_{5} x_{2} & -k_{5} x_{1} & 2 k_{2} x_{3} \\
-k_{5} x_{2} & -k_{3}-k_{5} x_{1} & k_{4} \\
2 k_{1}+3 k_{5} x_{2} & k_{3}+3 k_{5} x_{1} & -4 k_{2} x_{3}-k_{4}
\end{array}\right) .
$$

It has been proven by SNA that there is at least one saddle-node in the system. Bistability has been found numerically for a certain range of parameters. The question is now to determine this parameter range more precisely. Taking into account the conservation relation of total adsorption sites, the threedimensional system becomes two-dimensional with the general form of the characteristic polynomial $c p=a_{2} \lambda^{2}+a_{1} \lambda+a_{0}$ :

$$
\begin{aligned}
c p= & \lambda^{2}+\left(k_{4}+4 k_{2} x_{3}+k_{5} x_{2}+k_{3}+k_{1}+k_{5} x_{1}\right) \lambda \\
& -2 k_{5} x_{2} k_{2} x_{3}+4 k_{5} x_{1} k_{2} x_{3}+k_{5} x_{2} k_{4}+k_{5} x_{2} k_{3} \\
& +4 k_{3} k_{2} x_{3}-2 k_{5} x_{1} k_{4}+k_{1} k_{5} x_{1}+k_{1} k_{4}+k_{1} k_{3} .
\end{aligned}
$$

Bistability occurs only if $a_{0}$ has at least two sign changes [1]. It is a polynomial in the concentrations $x$. Substituting here the stationary concentrations, no conclusions concerning the number of sign changes can be made because of the complexity of the expressions. Hence, quotient rings are used in order to simplify $a_{0}$.

\subsection{Theory of Quotient Rings}

Definition 1. Let $k\left[x_{1}, \ldots, x_{n}\right]$ be a polynomial ring, let $I \subset k\left[x_{1}, \ldots, x_{n}\right]$ be an ideal, and let $f, g \in$ $k\left[x_{1}, \ldots, x_{n}\right]$. We say $f$ and $g$ are congruent modulo $I$, written

$$
f \equiv g \bmod I
$$

if $f-g \in I$. Then congruence modulo $I$ is an equivalence relation on $k\left[x_{1}, \ldots, x_{n}\right]$. (An equivalence relation on a set $S$ partitions $S$ into a collection of disjoint subsets called equivalence classes.) For any $f \in k\left[x_{1}, \ldots, x_{n}\right]$, the class $f$ is the set

$$
[f]=\left\{g \in k\left[x_{1}, \ldots, x_{n}\right]: g \equiv f \bmod I\right\} .
$$

Definition 2. The quotient of $k\left[x_{1}, \ldots, x_{n}\right]$ modulo $I$, written $k\left[x_{1}, \ldots, x_{n}\right] / I$, is the set of equivalence classes for congruence modulo $I$ :

$$
k\left[x_{1}, \ldots, x_{n}\right] / I=\left\{[f]: \in k\left[x_{1}, \ldots, x_{n}\right]\right\} .
$$

A special case occurs, if we take a Groebner basis $G B_{I}$ for the ideal $I$ and a polynomial $f \in k\left[x_{1}, \ldots, x_{n}\right]$. Fixing a monomial ordering on $k\left[x_{1}, \ldots, x_{n}\right]$ and applying the division algorithm 
(Buchberger algorithm), the remainder $r$ (normal form) satisfies $f=q+r$, where $q \in I$. Hence, $f-r=q \in I$, so $f \equiv r \bmod I$. In this case the remainder, i.e. the normal form, becomes unique. [2] Note that the generation of the normal form requires a monomial ordering. We will choose the pure lexicographic order again.

Example 1. Let $I=\left\langle z_{1}-k_{1} x_{1}, z_{2}-k_{2} x_{1} x_{2}, z_{3}-k_{1} x_{2}\right\rangle$ be an ideal and let $G B_{I}=\left\{g b_{1}=\right.$ $\left.k_{2} z_{1} z_{3}-k_{1}^{2} z_{2}, g b_{2}=k_{1} x_{2}-z_{3}, g b_{3}=k_{1} x_{1}-z_{1}\right\}$ be its corresponding Groebner basis with respect to the pure lexicographic order $\left\{x_{1}, x_{2}, z_{1}, z_{2}, z_{3}\right\}$. Further let $f=k_{1} k_{2} x_{1}+k_{1} x_{1} x_{2}-k_{2} x_{2}^{2} \in k\left[x_{1}, \ldots, x_{n}\right]$ be a polynomial the normal form of which is of interest. Then $f-r$ can be represented as a sum of multiples of the elements of $G B_{I}$

$$
f-r=c_{1} g b_{1}+c_{2} g b_{2}+c_{3} g b_{3},
$$

where

$$
c_{1}=\frac{1}{k_{1} k_{2}}, \quad c_{2}=x_{1}-\frac{k_{2} x_{2}}{k_{1}}-\frac{z_{3} k_{2}}{k_{1}^{2}}, \quad c_{3}=k_{2}+\frac{z_{3}}{k_{1}},
$$

and

$$
r=\operatorname{normal} f\left(f, G B_{I}\right)=z_{1} k_{2}+\frac{k_{1} z_{2}}{k_{2}}-\frac{z_{3}^{2} k_{2}}{k_{1}^{2}} .
$$

One sees that this method eliminates the variables $x_{1}$ and $x_{2}$. This concept will be applied to the coefficient $a_{0}$ of the characteristic polynomial in order to obtain the number of sign changes.

\subsection{Normal Forms}

In the following analysis two quotient rings and normal forms of $a_{0}$ will be considered. The first one is induced by the ideal $J(7)$ with its Groebner basis $G B_{J}(8)$. The second one is induced by the ideal

$$
\begin{aligned}
I= & \left\langle v_{1}-j_{3}, v_{2}-j_{2}-j_{3}, v_{3}-j_{1}, v_{4}-j_{1}-j_{2},\right. \\
& \left.v_{5}-j_{2},-k_{2} v_{4}^{2}+k_{4}{ }^{2} v_{2}, v_{3} v_{1} k_{5}-k_{3} k_{1} v_{5}\right\rangle,
\end{aligned}
$$

see (6), with the Groebner basis

$$
\begin{aligned}
G B_{I}= & \left\{2 k_{5} j_{1}{ }^{2} k_{2} j_{2}+k_{2} j_{2}{ }^{2} k_{5} j_{1}+k_{5} j_{1}{ }^{3} k_{2}-k_{5} j_{1} k_{4}{ }^{2} j_{2}-k_{4}{ }^{2} k_{3} k_{1} j_{2},-2 k_{2} j_{1} j_{2}\right. \\
& -k_{2} j_{2}{ }^{2}-k_{2} j_{1}{ }^{2}+k_{4}{ }^{2} j_{3}+k_{4}{ }^{2} j_{2}, v_{5}-j_{2}, v_{4}-j_{1}-j_{2}, v_{3}-j_{1}, k_{4}{ }^{2} v_{2} \\
& \left.-2 k_{2} j_{1} j_{2}-k_{2} j_{2}{ }^{2}-k_{2} j_{1}{ }^{2}, v_{1} k_{4}{ }^{2}-2 k_{2} j_{1} j_{2}-k_{2} j_{2}{ }^{2}-k_{2} j_{1}{ }^{2}+k_{4}{ }^{2} j_{2}\right\}
\end{aligned}
$$

with respect to the pure lexicographic monomial order with variables ordering

$$
\left\{v_{1}, v_{2}, v_{3}, v_{4}, v_{5}, j_{3}, j_{2}, j_{1}\right\}
$$

These two ideals, $J$ and $I$, have in common that all of their elements vanish at steady state. This fact is trivial for the ideal $I$. The elements of the ideal $J$ vanish because the stationary reaction rates lie in the convex flux cone and in the toric variety. This has the effect that every polynomial, which is expanded with respect to the polynomials of the two Groebner bases, reduces to its normal form when it is evaluated at steady state. 
The normal form of the polynomial $a_{0}$ modulo the Groebner basis $G B_{J}$ is a polynomial in the reaction rates $v$. It reads

$$
\begin{aligned}
\operatorname{normal} f\left(a_{0}, G B_{J}\right)= & \frac{1}{k_{1} k_{3} k_{4}}\left(4 k_{3}{ }^{2} k_{2} k_{1} v_{4}+k_{1} k_{5} k_{4} k_{3} v_{3}+k_{1} k_{5} k_{4}{ }^{2} v_{3}\right. \\
& -2 k_{5} k_{2} k_{1} v_{4} v_{3}-2 k_{5} k_{4}{ }^{2} v_{1} k_{3}+k_{5} k_{4} k_{1} v_{1} k_{3} \\
& \left.+4 k_{5} k_{2} v_{4} v_{1} k_{3}+{k_{1}}^{2} k_{3}{ }^{2} k_{4}+k_{1}{ }^{2} k_{4}{ }^{2} k_{3}\right) .
\end{aligned}
$$

To evaluate this term at steady state, one just has to substitute the stationary flux vector in convex parameters $\mathbf{j}$. This has the advantage that one gets rid of the radical terms of the stationary concentrations. However, since three convex parameters are reintroduced, one can hardly find the bistability region in the space of kinetic parameters. Nevertheless, it is possible to eliminate some convex parameters, mapping the normal form normal $f\left(a_{0}, G B_{J}\right)$ into the second quotient ring which is induced by the ideal $I$. In this space one has to consider the normal form normal $f\left(a_{0}, G B_{J}\right)$ modulo the Groebner basis $G B_{I}$, normal $f$ (normal $\left.f\left(a_{0}, G B_{J}\right), G B_{I}\right)$

$$
\begin{aligned}
& \text { normal } f\left(\text { normal } f\left(a_{0}, G B_{J}\right), G B_{I}\right)= \\
& \frac{1}{k_{4}^{3} k_{1} k_{3}}\left(k_{1}{ }^{2} k_{3}{ }^{2} k_{4}{ }^{3}+k_{1}{ }^{2} k_{4}{ }^{4} k_{3}+4 k_{3}{ }^{2} k_{2} k_{1} k_{4}{ }^{2} j_{1}+k_{5} k_{4}{ }^{3} k_{1} k_{3} j_{1}\right. \\
& +k_{5} k_{4}{ }^{4} k_{1} j_{1}-2 k_{5} k_{2} k_{1} k_{4}{ }^{2} j_{1}{ }^{2}-2 k_{5} k_{4}{ }^{2} k_{3} k_{2} j_{1}{ }^{2}+k_{5} k_{4} k_{3} k_{1} k_{2} j_{1}{ }^{2} \\
& -8 k_{5} k_{2}{ }^{2} k_{3} j_{1}{ }^{3}+16 k_{3}{ }^{2} k_{2} k_{1} k_{4}{ }^{2} j_{2}+2 k_{5} k_{4}{ }^{4} k_{3} j_{2}-k_{5} k_{4}{ }^{3} k_{3} k_{1} j_{2} \\
& +4 k_{5} k_{2} k_{3} j_{1} k_{4}{ }^{2} j_{2}-2 k_{5} k_{2} k_{1} k_{4}{ }^{2} j_{2} j_{1}+2 k_{5} k_{4} k_{3} k_{1} k_{2} j_{1} j_{2} \\
& \left.-12 k_{5} k_{2}{ }^{2} k_{3} j_{2} j_{1}{ }^{2}-6 k_{5} k_{2} k_{3} j_{2}{ }^{2} k_{4}{ }^{2}+k_{5} k_{4} k_{3} k_{1} k_{2} j_{2}{ }^{2}+4 k_{5} k_{2}{ }^{2} k_{3} j_{2}{ }^{3}\right) .
\end{aligned}
$$

A sign change in this expression is equivalent to a sign change in the coefficient $a_{0}$. To account for bistability this expression must change sign at least twice.

It is now necessary to check the influence of the remaining convex parameters $j_{1}$ and $j_{2}$ on the number of sign changes of normal $f\left(\right.$ normal $\left.f\left(a_{0}, G B_{J}\right), G B_{I}\right)$. For very low values of $j_{1}$ the second extreme current is predominating. In the limit $j_{1} \rightarrow 0$ the expression for normal $f\left(\right.$ normal $\left.f\left(a_{0}, G B_{J}\right), G B_{I}\right)$ reads

$$
\begin{aligned}
& \lim _{j_{1} \rightarrow 0} \text { normal } f\left(\text { normal } f\left(a_{0}, G B_{J}\right), G B_{I}\right)= \\
& \frac{1}{k_{4}^{3} k_{1} k_{3}}\left(4 k_{5} k_{2}{ }^{2} k_{3} j_{2}{ }^{3}+\left(-6 k_{5} k_{4}{ }^{2} k_{3} k_{2}+k_{5} k_{3} k_{4} k_{1} k_{2}\right) j_{2}{ }^{2}\right. \\
& \left.+\left(16 k_{3}{ }^{2} k_{2} k_{1} k_{4}{ }^{2}+2 k_{5} k_{4}{ }^{4} k_{3}-k_{5} k_{4}{ }^{3} k_{1} k_{3}\right) j_{2}+k_{1}{ }^{2} k_{3}{ }^{2} k_{4}{ }^{3}+k_{1}{ }^{2} k_{4}{ }^{4} k_{3}\right) .
\end{aligned}
$$

For very low and very high values of $j_{2}$ this expression is positive for all values of kinetic parameters. The system is stable. Looking closer at the coefficients of the expression as a polynomial in $j_{2}$

$$
\text { normalf }\left(\text { normalf }\left(a_{0}, G B_{J}\right), G B_{I}\right)=c_{3} j_{2}^{3}+c_{2} j_{2}^{2}+c_{1} j_{2}+c_{0}
$$

it becomes clear from Descartes' rule of sign that there may be at most two sign changes depending on the choice of parameters.

$$
\begin{aligned}
& c_{1}=16 k_{3}^{2} k_{2} k_{1} k_{4}^{2}+k_{4}^{3} k_{5} k_{3}\left(2 k_{4}-k_{1}\right) \\
& c_{2}=k_{5} k_{4} k_{3} k_{2}\left(-6 k_{4}+k_{1}\right) .
\end{aligned}
$$

Note that the coefficients $c_{3}$ and $c_{0}$ are always positive. Most important is the relation between $k_{4}$ and $k_{1}$. Consequently two cases must be distinguished: 

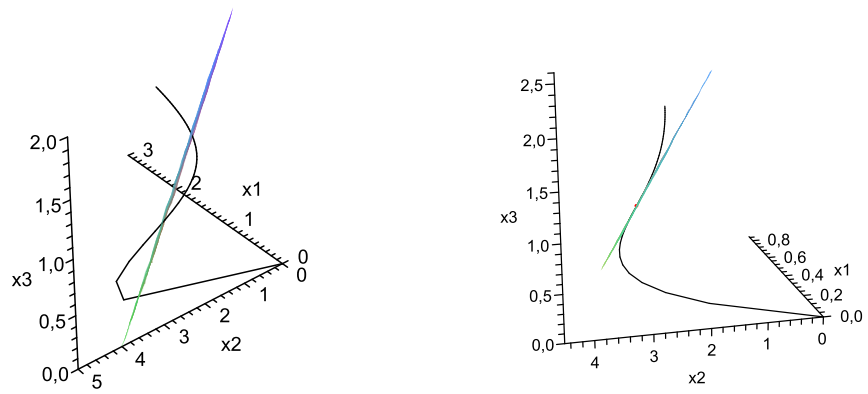

Figure 2: LH mechanism. (Left) Bistability is given for $k_{1}=k_{3}=0.25, k_{2}=k_{4}=k_{5}=4, c=4$. The curve of the stationary concentration intersects the plane of the conservation relation three times. (Right) For $k_{1}=6, k_{2}=k_{4}=k_{5}=4, k_{3}=0.417, c=4.5508$ the two saddle-node bifurcations come together in a cusp.

$k_{1}<6 k_{4}: \quad c_{2}$ is negative. $a_{0}$ and normal $f\left(\right.$ normal $\left.f\left(a_{0}, G B_{J}\right), G B_{I}\right)$ can have two sign changes. Bistability is possible.

$k_{1}>2 k_{4}$ : For high values of $k_{3}$ both coefficients $c_{1}$ and $c_{2}$ are positive. It follows that $a_{0}$ and normal $f$ (normal $\left.f\left(a_{0}, G B_{J}\right), G B_{I}\right)$ have no sign change for any constellation of kinetic parameters. The system is monostable. For $16 k_{2} k_{3}<k_{4} k_{5}$ and low values of $k_{3}$ the coefficient $c_{1}$ becomes negative, but this only leads with $c_{2}<0$ to a sign change in normal $f\left(\right.$ normal $\left.f\left(a_{0}, G B_{J}\right), G B_{I}\right)$. A second region of bistability can be excluded. The condition that $c_{2}$ is positive before $c_{1}$ becomes negative (with increasing $\left.k_{1}\right)$ requires a parameter constellation such that normal $f\left(\right.$ normal $\left.f\left(a_{0}, G B_{J}\right), G B_{I}\right)$ can not have two sign changes since the other coefficients grow faster (with increasing $k_{1}$ or $j_{2}$ ) than $c_{1}$.

Hence, bistability is assured by $k_{4}>k_{1}$, see left in Fig. 2. In Fig. 2 (right), a parameter constellation is given for a cusp - for higher values of $k_{3}$ the system becomes monostable. In both Figures we use the conservation relation (3) as a plane in the concentration space given by $c=$ $2 x_{1}+x_{2}+x_{3}$. For very low values of $j_{2}$ the first extreme current predominates. In the limit $j_{2} \rightarrow 0$ the normalf $\left(\right.$ normalf $\left.\left(a_{0}, G B b_{J}\right), G B_{I}\right)$ reads

$$
\begin{aligned}
& \lim _{j_{2} \rightarrow 0} \text { normal } f\left(\text { normal } f\left(a_{0}, G B_{J}\right), G B_{I}\right)= \\
& \frac{1}{k_{4}^{3} k_{3} k_{1}}\left(-8 k_{5} k_{3}{k_{2}}^{2} j_{1}{ }^{3}+\left(-2 k_{5} k_{2} k_{1} k_{4}{ }^{2}-2 k_{5} k_{2} k_{3} k_{4}{ }^{2}+k_{5} k_{4} k_{3} k_{1} k_{2}\right) j_{1}{ }^{2}\right. \\
& \left.+\left(4 k_{3}{ }^{2} k_{2} k_{1} k_{4}{ }^{2}+k_{5} k_{4}{ }^{3} k_{1} k_{3}+k_{1} k_{5} k_{4}{ }^{4}\right) j_{1}+{k_{3}}^{2} k_{1}{ }^{2} k_{4}{ }^{3}+k_{1}{ }^{2} k_{4}{ }^{4} k_{3}\right)
\end{aligned}
$$

Evidently, this expression can have at most one sign change. It follows that $a_{0}$ may have at most one sign change for all values of kinetic parameters and the system cannot become bistable for high values of $j_{1}$. It is known from $S N A$ that, in fact, the first and third extreme currents are stable equilibrium currents which by themselves exhibit a zero eigenvalue that is not relevant for the stability problem.

Remark Generating a normal form of $a_{0}$ modulo $G B$ requires a variables ordering for both $G B$ and the normal form. Assumed that the variables ordering is the same for both there are six possibilities concerning the convex parameter $j_{i}$. With variables ordering $\left\{v_{1}, v_{2}, v_{3}, v_{4}, v_{5}, j_{1}, j_{2}, j_{3}\right\}$ and $\left\{v_{1}, v_{2}, v_{3}, v_{4}, v_{5}, j_{1}, j_{3}, j_{2}\right\}$ no convex parameter is eliminated. $\left\{v_{1}, v_{2}, v_{3}, v_{4}, v_{5}, j_{2}, j_{1}, j_{3}\right\}$ and $\left\{v_{1}, v_{2}, v_{3}, v_{4}, v_{5}, j_{2}, j_{3}, j_{1}\right\}$ lead to normal forms without $j_{2}$. The ordering $\left\{v_{1}, v_{2}, v_{3}, v_{4}, v_{5}, j_{3}, j_{1}, j_{2}\right\}$ 
or $\left\{v_{1}, v_{2}, v_{3}, v_{4}, v_{5}, j_{3}, j_{2}, j_{1}\right\}$ eliminates $j_{3}$ and the resulting normal forms are the same. Of course there are more possibilities, but this problem is discussed in section 5 .

\section{Carbon Monoxide Oxidation}

As a real reaction system the $C O$ oxidation on platinum exemplifies the abstract LH mechanism. Both LH mechanism and $C O$ oxidation have been studied in detail in [6]. The $C O$ oxidation consists of dissociative adsorption of oxygen (which requires 2 adjacent sites), adsorption and desorption of $C O$ ( 1 site), surface reaction and a step taking asymmetric inhibition into account, i. e. $C O$ can adsorb on an $O$-covered surface, where it reacts practically immediately; the desorption of oxygen is neglected.

Denoting $O_{a d}, C O_{a d}$, and $*$ with $X_{1}, X_{2}$, and $X_{3}$, respectively, leaving out the nonessential gaseous species and neglecting the very short coexistence of $\left[\mathrm{O}_{a d}+\mathrm{CO}_{a d}\right]$ in the last process, the reactions of the $C O$ oxidation mechanism become the pseudoreactions

$$
\begin{array}{rllrll}
2 *+O_{2} & \longrightarrow & 2 O_{a d} & 2 X_{3} & \stackrel{k_{2}}{\longrightarrow} & 2 X_{1} \\
C O_{a d} & \longrightarrow & *+C O & X_{2} & \stackrel{k_{3}}{\longrightarrow} & X_{3} \\
*+C O & \longrightarrow & C O_{a d} & X_{3} & \stackrel{k_{4}}{\longrightarrow} & X_{2} \\
O_{a d}+C O_{a d} & \longrightarrow & 2 *+C O_{2} & X_{1}+X_{2} & \stackrel{k_{5}}{\longrightarrow} & 2 X_{3} \\
O_{a d}+C O \rightarrow & {\left[O_{a d}+C O_{a d}\right]} & \rightarrow *+C O_{2} & X_{1} & \stackrel{k_{1}}{\longrightarrow} & X_{3}
\end{array}
$$

The corresponding network diagram is shown in Fig. 3 on the left. Note that the order of the rate constants is adapted to the abstract model of the LH mechanism, see section 2.

The kinetic equations are

$$
\begin{aligned}
& \dot{x_{1}}=-k_{1} x_{1}+2 k_{2} x_{3}{ }^{2}-k_{5} x_{1} x_{2} \\
& \dot{x_{2}}=-k_{3} x_{2}+k_{4} x_{3}-k_{5} x_{1} x_{2} \\
& \dot{x_{3}}=k_{1} x_{1}-2 k_{2} x_{3}{ }^{2}+k_{3} x_{2}-k_{4} x_{3}+2 k_{5} x_{1} x_{2}
\end{aligned}
$$

with the conservation relation

$$
x_{1}+x_{2}+x_{3}=\text { const } \text {. }
$$

The stoichiometric matrix $N$, the flux vector $v$, and the kinetic matrix $\kappa$ are given by

$$
N=\left(\begin{array}{ccccc}
-1 & 2 & 0 & 0 & -1 \\
0 & 0 & -1 & 1 & -1 \\
1 & -2 & 1 & -1 & 2
\end{array}\right), \quad v=\left(\begin{array}{c}
k_{1} x_{1} \\
k_{2} x_{3}{ }^{2} \\
k_{3} x_{2} \\
k_{4} x_{3} \\
k_{5} x_{1} x_{2}
\end{array}\right) \quad \kappa=\left(\begin{array}{ccccc}
1 & 0 & 0 & 0 & 1 \\
0 & 0 & 1 & 0 & 1 \\
0 & 2 & 0 & 1 & 0
\end{array}\right)
$$

Using (4), the extreme currents which span the cone and the flux vector with convex parameters are obtained, see (37). The extreme currents as diagrams are shown in Fig. 3.

$$
E_{1}=\left(\begin{array}{l}
0 \\
0 \\
1 \\
1 \\
0
\end{array}\right), E_{2}=\left(\begin{array}{l}
0 \\
1 \\
0 \\
2 \\
2
\end{array}\right), E_{3}=\left(\begin{array}{l}
2 \\
1 \\
0 \\
0 \\
0
\end{array}\right), \quad v(j)=\left(\begin{array}{c}
2 j_{3} \\
j_{2}+j_{3} \\
j_{1} \\
j_{1}+2 j_{2} \\
2 j_{2}
\end{array}\right) .
$$

Following section 2.2 we use the Groebner basis of the toric variety which is generated by the reaction rates $v_{j}-v_{j}(x, k)$ to separate the reaction rates from the concentrations. The resulting deformed toric 


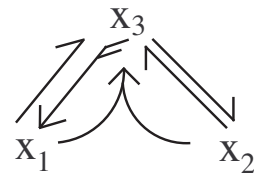

$\mathrm{D}_{\mathrm{N}}$

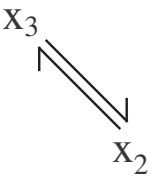

$E_{1}$

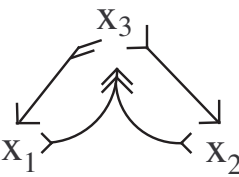

E2

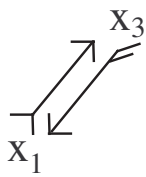

$\mathrm{E}_{3}$

Figure 3: The network diagram $D_{N}$ of the $C O$ oxidation and the extreme currents $E_{i} . X_{1}$ represents $O, X_{2}$ is $C O$, and a vacant site is denoted by $X_{3} . E_{1}$ and $E_{3}$ are an equilibrium respectively pseudoequilibrium current and therefore stable, $E_{2}$ is unstable.

variety - the elements of the Groebner basis which are independent of the concentrations - is intersected with the cone, see (10). Subsequently, the convex parameters $j_{i}$ can be solved for and via the Hermite normal form algorithm (see section 2.2) the parameterized set of the stationary solutions are obtained:

$$
x(j)=\left(\begin{array}{c}
2 \frac{j}{k_{1}} \\
1 / 2 \frac{\left(j k_{5} k_{4}+\sqrt{j^{2} k_{5}{ }^{2} k_{4}{ }^{2}+16 k_{2} k_{3} k_{1} j^{2} k_{5}+16 k_{2} j^{3} k_{5}{ }^{2}+4 j k_{2} k_{3}{ }^{2} k_{1}{ }^{2}}\right) k_{1} k_{4}}{k_{2}\left(4 j k_{3} k_{1} k_{5}+4 j^{2} k_{5}{ }^{2}+k_{3}{ }^{2} k_{1}{ }^{2}\right)} \\
1 / 2 \frac{j k_{5} k_{4}+\sqrt{j\left(j k_{5}{ }^{2} k_{4}{ }^{2}+16 k_{2} k_{3} k_{1} j k_{5}+16 k_{2} j^{2} k_{5}{ }^{2}+4 k_{2} k_{3}{ }^{2} k_{1}{ }^{2}\right)}}{\left(k_{3} k_{1}+2 k_{5} j\right) k_{2}}
\end{array}\right)
$$

\subsection{Stability Analysis}

Since the radical expressions of the stationary concentrations make the stability analysis via the Jacobian matrix,

$$
\operatorname{Jac}(v)=\left(\begin{array}{ccc}
-k_{1}-k_{5} x_{2} & -k_{5} x_{1} & 4 k_{2} x_{3} \\
-k_{5} x_{2} & -k_{3}-k_{5} x_{1} & k_{4} \\
k_{1}+2 k_{5} x_{2} & k_{3}+2 k_{5} x_{1} & -4 k_{2} x_{3}-k_{4}
\end{array}\right),
$$

difficult, the concept of quotient rings is used to simplify the coefficient $a_{0}$ of the characteristic polynomial $c p=\lambda^{2}+a_{1} \lambda+a_{0}$ :

$$
\begin{aligned}
c p= & \lambda^{2}+\left(k_{5} x_{1}+k_{5} x_{2}+4 k_{2} x_{3}+k_{3}+k_{1}+k_{4}\right) \lambda \\
& +k_{5} x_{2} k_{3}+k_{5} x_{2} k_{4}+k_{1} k_{3}+4 k_{3} k_{2} x_{3}-k_{5} x_{1} k_{4} \\
& +k_{1} k_{4}+4 k_{5} x_{1} k_{2} x_{3}+k_{1} k_{5} x_{1}-4 k_{5} x_{2} k_{2} x_{3} .
\end{aligned}
$$

For bistability at least two sign changes in the coefficient $a_{0}$ are required. These sign changes are investigated by the normal form of $a_{0}$

$$
\begin{aligned}
& \text { normal } f\left(\text { normal } f\left(a_{0}, g b_{J}\right), g b_{I}\right)= \\
& \frac{1}{k_{4}^{3} k_{1} k_{3}}\left(k_{1}{ }^{2} k_{3}{ }^{2} k_{4}{ }^{3}+k_{1}{ }^{2} k_{4}{ }^{4} k_{3}+4 k_{3}{ }^{2} k_{2} k_{1} k_{4}{ }^{2} j_{1}+k_{5} k_{4}{ }^{3} k_{1} k_{3} j_{1}+k_{5} k_{4}{ }^{4} k_{1} j_{1}\right. \\
& -4 k_{5} k_{2} k_{1} k_{4}{ }^{2} j_{1}{ }^{2}-2 k_{5} k_{4}{ }^{2} k_{3} k_{2} j_{1}{ }^{2}+2 k_{5} k_{4} k_{3} k_{1} k_{2} j_{1}{ }^{2}-16 k_{5} k_{2}{ }^{2} k_{3} j_{1}{ }^{3} \\
& +32 k_{3}{ }^{2} k_{2} k_{1} k_{4}{ }^{2} j_{2}+2 k_{5} k_{4}{ }^{4} k_{3} j_{2}-2 k_{5} k_{4}{ }^{3} k_{3} k_{1} j_{2}+8 k_{5} k_{2} k_{3} j_{1} k_{4}{ }^{2} j_{2} \\
& -8 k_{5} k_{2} k_{1} k_{4}{ }^{2} j_{2} j_{1}+8 k_{5} k_{4} k_{3} k_{1} k_{2} j_{1} j_{2}-48 k_{5} k_{2}{ }^{2} k_{3} j_{2} j_{1}{ }^{2}-24 k_{5} k_{2} k_{3} j_{2}{ }^{2} k_{4}{ }^{2} \\
& \left.+8 k_{5} k_{4} k_{3} k_{1} k_{2} j_{2}{ }^{2}+64 k_{5} k_{2}{ }^{2} k_{3} j_{2}{ }^{3}\right) .
\end{aligned}
$$


As in section 3.3 we examine the influence of the convex parameters on the number of sign changes of the normal form (41). Note the remaining convex parameters are the same as in (30): $j_{1}$ and $j_{2}$. For very small $j_{1}$, one obtains

$$
\begin{aligned}
& \lim _{j_{1} \rightarrow 0} \text { normal } f\left(\text { normal } f\left(a_{0}, G B_{J}\right), G B_{I}\right)= \\
& \frac{1}{k_{4}^{3} k_{1} k_{3}}\left(64 k_{3} k_{5} k_{2}{ }^{2} j_{2}{ }^{3}+\left(-24 k_{5} k_{2} k_{3} k_{4}{ }^{2}+8 k_{5} k_{4} k_{3} k_{1} k_{2}\right) j_{2}{ }^{2}\right. \\
& +\left(32 k_{3}{ }^{2} k_{2} k_{1} k_{4}{ }^{2}+2 k_{5} k_{4}{ }^{4} k_{3}-2 k_{5} k_{4}{ }^{3} k_{3} k_{1}\right) j_{2} \\
& \left.+k_{1}{ }^{2} k_{3}{ }^{2} k_{4}{ }^{3}+k_{1}{ }^{2} k_{4}{ }^{4} k_{3}\right) .
\end{aligned}
$$

The coefficients are

$$
\begin{aligned}
c_{0} & =k_{1}{ }^{2} k_{3}{ }^{2} k_{4}{ }^{3}+k_{1}{ }^{2} k_{4}{ }^{4} k_{3} \\
c_{1} & =32 k_{3}{ }^{2} k_{2} k_{1} k_{4}{ }^{2}+2 k_{5} k_{4}{ }^{3} k_{3}\left(k_{4}-k_{1}\right) \\
c_{2} & =8 k_{5} k_{4} k_{3} k_{2}\left(k_{1}-3 k_{4}\right) \\
c_{3} & =64 k_{3} k_{5} k_{2}{ }^{2} .
\end{aligned}
$$

$c_{0}$ and $c_{3}$ are always positive. $c_{1}$ and $c_{2}$ are similar to the coefficients in (32). We know that a sign change in normal $f$ (normal $\left.f\left(a_{0}, G B_{J}\right), G B_{I}\right)$ is given if either $c_{2}$ is negative or both coefficients $c_{1}$ and $c_{2}$ are negative and bistability for high values of $k_{1}$ can be excluded. Additionally, this situation is physically impossible since the two processes (reactive removal of adsorbed $O$ and adsorption of $C O$ ) are both (practically) equal to the impingement rate of $C O$, hence $k_{1} \lesssim k_{4}$. Consequently, the conditions for bistability have to be obtained from $c_{2}$, namely $k_{1}<3 k_{4}$.

The parameter constellation is approximately the same as before to show bistability and the cusp, see Fig. 4.

The influence of a small second extreme current can be derived by the expression

$$
\begin{aligned}
& \lim _{j_{2} \rightarrow 0} \text { normal } f\left(\text { normal } f\left(a_{0}, G B_{J}\right), G B_{I}\right)= \\
& \frac{1}{k_{4}^{3} k_{1} k_{3}}\left(-16 k_{3} k_{5}{k_{2}}^{2} j_{1}{ }^{3}+\left(-4 k_{5} k_{2} k_{1}{k_{4}}^{2}-2 k_{5} k_{2} k_{3} k_{4}{ }^{2}+2 k_{5} k_{4} k_{3} k_{1} k_{2}\right) j_{1}{ }^{2}\right. \\
& \left.+\left(4 k_{3}{ }^{2} k_{2} k_{1} k_{4}{ }^{2}+k_{5} k_{4}{ }^{3} k_{1} k_{3}+k_{1} k_{5} k_{4}{ }^{4}\right) j_{1}+\left(k_{1}{ }^{2} k_{3}{ }^{2} k_{4}{ }^{3}+k_{1}{ }^{2} k_{4}{ }^{4} k_{3}\right)\right),
\end{aligned}
$$

which can have at most one sign change. Hence, the system cannot become bistable for high values of $j_{1}$.

\section{Discussion}

We analytically showed the existence of bistability in typical LH mechanisms. Since the solutions resulted in complicated expressions, a simplification via quotient rings was introduced successfully. The relevant coefficient of the characteristic polynomial of the Jacobian matrix was simplified by substituting variables which can be partially eliminated. This led to polynomial expressions, normal forms, a sign change in the coefficients of which could be found and parameter constellations were given to show bistability and a cusp.

The problem of the concept of quotient rings is to find the best variables ordering determining the convex parameter $j$ which is possibly eliminated concerning the resulting normal forms. Three convex parameters means there are six possibilities of ordering assuming the ordering is preserved for the corresponding Groebner basis and the resulting normal form. Since it is known from SNA that the second extreme current is unstable, the resulting normal form has to contain this extreme current. Hence, orderings which eliminate $j_{2}$ can be neglected since the resulting normal forms have at most only one 

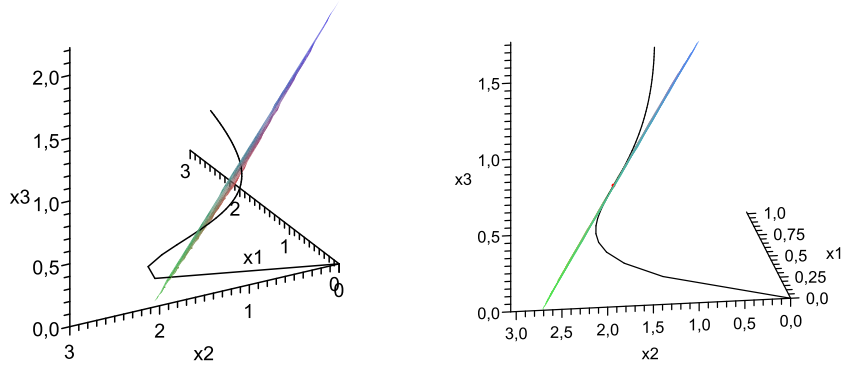

Figure 4: $C O$ oxidation. (Left) Bistability results for $k_{1}=0.5, k_{3}=0.25, k_{2}=k_{4}=k_{5}=4, c=2.1$. The curve of the stationary concentration intersects the plane of the conservation relation three times. (Right) For $k_{1}=6, k_{2}=4, k_{3}=0.4136, k_{4}=k_{5}=4, c=2.71856$ a cusp occurs.

zero, excluding bistability. Several orderings do not lead to an elimination of any convex parameter and complicate the stability analysis. Different variables ordering for Groebner basis and resulting normal form were not tested. In this context it should be helpful to use not the affine toric ideal $J$, but its homogeneous form, a projective toric ideal.

It has been shown that the application of the projective toric ideal reduces the number of normal forms using all possible variables orderings [2]. These expressions enable an investigation of the individual influences of the kinetic parameters. In our case the resulting projective toric variety is a plane in the flux cone. If it is cut by a normalizing plane, it becomes a curve. This means that the curve of stationary fluxes is projected onto a plane $1-\sum_{i=1} j_{i}$. The stability analysis for the steady states on this curve is equivalent to the stability analysis for the whole flux cone. This concept including projective varieties and quotient rings may offer a more detailed and more easily applicable stability analysis and will be the subject of further investigations.

\section{Acknoledgement}

The authors commemorate Karin Gatermann who introduced us to algebraic geometry and developed excellent ideas for applications in chemistry. 


\section{References}

[1] B. L. Clarke, Adv. Chem. Phys. 43, 1 (1980).

[2] D. Cox, J. Little and D. O'Shea, Ideals, Varieties, and Algorithms, Undergraduate Texts in Mathematics, Springer, New York, 1992.

[3] G. Craciun and M. Feinberg, SIAM J. Appl. Math. 65, 5 (2005).

[4] G. Craciun and M. Feinberg, SIAM J. Appl. Math. 66, 4 (2006).

[5] M. Domijan and M. Kirkilionis, Journal of Mathematical Biology (2008) DOI 10.1007/s00285-0080234-7.

[6] M. Eiswirth, J. Bürger, P. Strasser, G. Ertl, J. Phys. Chem. 100, 49 (1996).

[7] V. Fairén and B. Hernández-Bermejo, J. Phys. Chem. 100, 19023 (1996).

[8] M. Feinberg and D. Terman, Arch. Rational Mech. Anal. 116, 35 (1991).

[9] K. Gatermann, in: Symbolic Computation: Solving Equations in Algebra, Geometry, and Engineering, volume 286 of Contemporary Mathematics, American Mathematical Society 2001, p. 53.

[10] K. Gatermann, M. Eiswirth and A. Sensse, J. Symbolic Comput. 40, 1361 (2005).

[11] K. Gatermann and B. Huber, J. Symbolic Comput. 33, 275 (2002).

[12] A. Sensse, Convex and toric geometry to analyze complex dynamics in chemical reaction systems, Otto-von-Guericke-Universität, Magdeburg, PhD Thesis, 2005.

[13] A. Sensse, K. Gatermann and M. Eiswirth, Journal of Electroanalytical Chemistry 577, 35 (2005). 